\title{
Cancer Imaging and Brain Tumor Diagnosis
}

\section{Hamidreza Shirzadfar*, Samin Riahi and Mahsa Sadat Ghaziasgar}

Department of Biomedical Engineering, Sheikhbahaee University, Baharestan, Isfahan, Iran

\section{Editorial}

The human brain is a central nervous system including varied parts which everyone has different tasks as they have a close connection with each other. The human brain consists of two sections called Central nervous and Peripheral nervous system. The nervous part is the center of automatic or involuntary activities and peripheral part is composed of the nerves that connect the different parts of the body to the nervous system. The nervous system or neural system coordinates the muscles activities and monitors the various organs in the animals' body and causes pauses in entrances related to different senses, therefore the nervous system is differentiated and evolved for coordinating between cells' activities and various organs of the body with its special structure and function. It is different attributes are impressionable against the external stimulus and creating a nervous stream that shows the effect of stimuli, transmitting signals from one point of the system to another and finally, from one nervous unit to another unit. The nervous system is divided into two different parts anatomically including:

A) A Central nervous system that consisting of the brain and spinal cord is encased within a cylindrical shell called a skull and spinal cord.

B) The peripheral nervous system includes 12 pairs of nerve fibers of the brain and 21 pairs of nerve fibers of the spinal cord that connect the brain and spinal cord to the other parts of the body.

By collecting of the cancer cells and destroying cells of healthy tissues, a mass named Tumor creates. The cancer cells appeared from natural cells from a genetic mutation of the cell is presented in Figure 1.

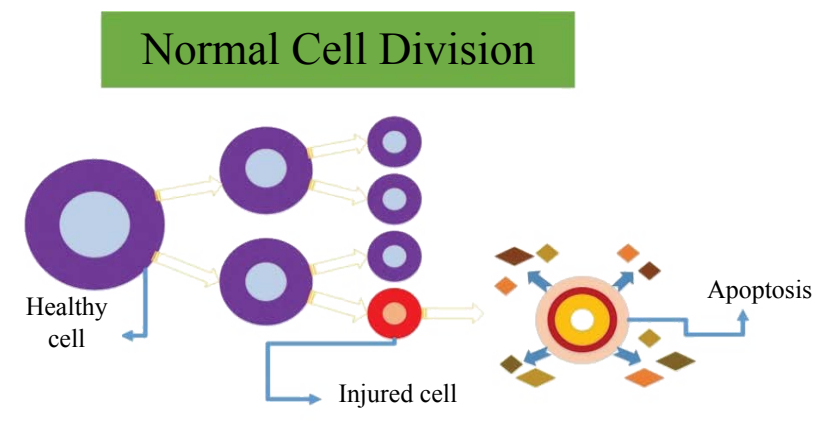

Cancer Cell Division

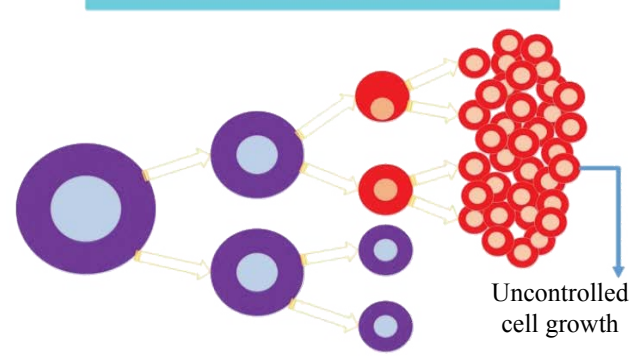

Figure 1: Cell division in normal and cancer cells.
A brain tumor is abnormal mass growth in the brain, or in other words, is a kind of tumor inside a hard and solid skull and or is a tumor within the brain or central canal of the spinal cord.

Due to their aggressive and spreading nature in the limited space of the skull, these types of tumor are life-threatening inherently and seriously but they are not fatal and mortal constantly.

The brain tumors can inherently be cancerous (malignant) or noncancerous (benign) [1]. However, the definition of malignant and benign neoplasm in the brain differs from the definitions which normally in other types of cancerous or non-cancerous tumors involving other parts of the body used. The level of threating of a tumor depends on a combination of various factors such as the type of tumor, location and size of the tumor and how to extend it. Since the brain is covered completely by the skull, rapid and precocious diagnosis of the brain tumor is possible only if the clinical utility and appropriate diagnostic tools that are well recognized the internal cavity of the skull, available and quickly be applied. But the brain tumor diagnosis normally occurs in the progression of the disease and when the tumor existing has been caused an unutterable indication of signs and symptoms in patients. The classification of brain tumors are:

A) Regarding place and origin of neoplasms [2-3]: A-1) Primary brain tumor: primary brain tumors (true) taken the root of the neuroepithelial tissue, and usually appear in the posterior cranial fossa in children and in 3/2 anterior from cerebral hemispheres in adults, although this type of brain tumor can affect in a part of the brain. There are various types of primary brain tumors that named according to the cells type and the area of the brain where they start to grow. A-2) Secondary brain tumor: secondary brain tumors are the same metastatic tumors that their primary origins are from the primary cancers related to other parts of the body and spread into the internal space range of the skull. This means that cancer cells penetrate out from the primary tumor that its origin is a tumor in the other organ, and then it enters the lymphatic system and blood vessels. These cells, then through the blood circulatory system will be widespread and settle in the brain. Then, these cells will continue to growing and divide irregularly turn into another aggressive neoplasm that is of the same material of the primary cancer tissue.

B) Regarding the treatment of Neoplasms [4]: Brain tumors or intracranial neoplasms can have the nature of cancerous (malignant) or noncancerous (benign). The definition of malignant or benign neoplasms of the brain differs from other parts of the body.

*Corresponding author: Hamidreza Shirzadfar, Department of Biomedical Engineering, Sheikhbahaee University, Baharestan, Isfahan, Iran, Tel: +983136530125; E-mail: h.shirzadfar@shbu.ac.ir

Received January 30, 2017; Accepted January 31, 2017; Published February 06, 2017

Citation: Shirzadfar H, Riahi S, Ghaziasgar MS (2017) Cancer Imaging and Brain Tumor Diagnosis. J Bioanal Biomed 9: e149. doi: 10.4172/1948-593X.1000e149

Copyright: (c) 2017 Shirzadfar H, et al. This is an open-access article distributed under the terms of the Creative Commons Attribution License, which permits unrestricted use, distribution, and reproduction in any medium, provided the original author and source are credited. 
Citation: Shirzadfar H, Riahi S, Ghaziasgar MS (2017) Cancer Imaging and Brain Tumor Diagnosis. J Bioanal Biomed 9: e149. doi: 10.4172/1948-593X.1000e149

By using sophisticated imaging technology can identify the location of the tumors. Medical imaging is the technology and the process of making images of the human body or body parts and functions for clinical purposes or medical science. The medical imaging is an interference of several branches of science, such as medical physics, biomedical engineering [5], biology, and optics [6]. Diagnostic tools include; Computed Tomography (CT), Positron Emission Tomography (PET scan), Magnetic Resonance spectroscopy (MRS), Magnetic Resonance Imaging (MRI), Angiogram, Ultrasound (US), X-ray imaging, Single-photon emission computed tomography (SPECT).

MRI is the most popular method to locate a tumor [7]. MRI is an examination technique that consists of creating precise images of a part of the body, using radio waves and a magnetic field. The images are reconstructed by a computer and interpreted by a radiologist. A MRI is performed using a large cylinder-shaped apparatus and this device is composed of a very powerful magnet. Sometimes, in during the examination a contrast agent is injected into a vein. It allows highlighting certain aspects of the brain, like the blood vessels and facilitates the interpretation of the images.

\section{References}

1. Reinsberg S, Scurr E, Brewster J, Payne G (2014) Magnetic resonance imaging in prostate cancer: the value of apparent diffusion coefficients for identifying malignant nodules. Br J Radiol 80: 90-95.

2. Hemmati HD, Nakano I, Lazareff JA, Masterman-Smith M, Geschwind DH, et al. (2003) Cancerous stem cells can arise from pediatric brain tumors. Proc Natl Acad Sci U S A 100: 15178-15183.

3. Shaw E, Scott C, Souhami L, Dinapoli R, Kline R, et al. (2000) Single dose radiosurgical treatment of recurrent previously irradiated primary brain tumors and brain metastases: final report of RTOG protocol 90-05. Int J Radiat Oncol Biol Phys 47: 291-298.

4. Acton QA (2013) Digestive System Neoplasms-Advances in Research and Treatment. (2013edn), Scholarly Editions, Atlanta, Gorgia, USA.

5. Shirzadfar H, Nadi M, Kourtiche D, Yamada S, Hauet T (2015) Needle-type GMR sensor to estimate the magnetic properties of diluted ferrofluid for biomedicine application. IRBM 36: 178-184.

6. Lin WC, Toms SA, Motamedi M, Jansen ED, Mahadevan-Jansen A (2000) Brain tumor demarcation using optical spectroscopy: an in vitro study. J Biomed Opt 5: 214-220.

7. Shirzadfar H (2014) Conception et réalisation d'un biocapteur à GMR pour la caractérisation de milieux biologiques. Université de Lorraine. 